\title{
Apoptotic effect of gambogic acid in esophageal squamous cell carcinoma cells via suppression of the NF- $\mathrm{KB}$ pathway
}

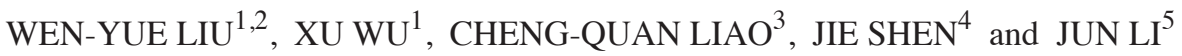 \\ ${ }^{1}$ Department of Thoracic and Cardiovascular Surgery, Nanfang Hospital, Southern Medical University, Guangzhou, \\ Guangdong 510515; Departments of ${ }^{2}$ Thoracic Surgery, ${ }^{3}$ Cardiovascular Surgery and ${ }^{4}$ Medical Records, \\ Yuebei People's Hospital, Shaoguan, Guangdong 512026; ${ }^{5}$ Department of Thoracic Surgery, \\ Third Affiliated Hospital of Southern Medical University, Guangzhou, \\ Guangdong 510630, P.R. China
}

Received February 14, 2015; Accepted March 9, 2016

DOI: $10.3892 / \mathrm{ol} .2016 .4437$

\begin{abstract}
Despite extensive investigations of therapeutic improvements for surgical techniques, chemotherapy and chemoradiotherapy, esophageal squamous cell carcinoma (ESCC) remains one of the most aggressive forms of cancer, and the prognosis for patients with advanced ESCC remains poor. Therefore, effective therapies are urgently required in order to improve the prognosis of patients with ESCC. TE-1 cells were treated with gambogic acid (GA), and then subjected to western blot analysis, TUNEL assay and caspase activity analysis. GA significantly induced apoptosis in ESCC TE-1 cells. In addition, the antitumor activity of GA was accompanied by the decreased expression of phosphorylated-protein kinase B (p-AKT) and nuclear factor of $\kappa$ light polypeptide gene enhancer in B-cells $1(\mathrm{NF}-\kappa \mathrm{B})$. The inhibition of protein kinase $\mathrm{B}(\mathrm{AKT})$ and $\mathrm{NF}-\kappa \mathrm{B}$ activation by chemical inhibitors augmented the apoptotic effect responses to GA in the TE-1 cells. The pan-caspase inhibitor z-VAD-fmk (zVAD) decreased GA-induced apoptosis. Furthermore, zVAD attenuated GA-induced growth inhibition in TE-1 cells. GA induced apoptosis in ESCC TE-1 via suppression of NF- $\kappa$ B pathway. The findings of the present study may provide a novel insight into ESCC treatment.
\end{abstract}

\section{Introduction}

Worldwide, esophageal cancer (EC) is the eighth most common cancer and sixth most common cause of cancer-associated

Correspondence to: $\mathrm{Dr} \mathrm{Xu} \mathrm{Wu}$, Department of Thoracic and Cardiovascular Surgery, Nanfang Hospital, Southern Medical University, 1838 North Guangzhou Da Dao Road, Guangzhou, Guangdong 510515, P.R. China

E-mail: xu_wu168@126.com

Key words: gambogic acid, esophageal squamous cell carcinoma, apoptosis, TE-1, nuclear factor of $\kappa$ light polypeptide gene enhancer in B-cells 1 mortality (1). It is generally diagnosed at a late stage of disease and has a poor prognosis, with a 5-year survival rate of $<10 \%$ (2). The majority of patients with EC in Asia, including China and Japan, have esophageal squamous cell carcinoma (ESCC), while the majority of patients in Western countries have esophageal adenocarcinoma (EAC) (3). Surgery and preoperative chemoradiotherapy are optional treatments for patients with resectable tumors to treat ESCC and EAC (4). The most commonly utilized chemotherapy agents are fluoropyrimidine, taxanes (paclitaxel or docetaxel) and platinum compounds. Although ESCC and EAC are responsive to chemotherapy, the response rates are low, particularly for patients with advanced diseases (5). Therefore, for ES patients, novel therapies are required.

Gambogic acid $\left(\mathrm{GA} ; \mathrm{C}_{38} \mathrm{H}_{44} \mathrm{O}_{8}\right.$; molecular weight, $628.75)$ is the major active ingredient in gamboges, and is a brownish orange dry resin secreted from Garcinia hanburyi, a plant that primarily grows in South China, Cambodia and Thailand (6). Gamboge resin has been used as a coloring material and in traditional Chinese medicine (TCM) for the treatment of human diseases. Previous studies have demonstrated that GA has anticancer effects and inhibits the growth of multiple types of human cancer cells in vitro and in vivo $(7,8)$. In animal tumor models and clinical trials, GA efficiently inhibits tumor growth with minimal side effects, with little toxicity on the immune and hemopoietic systems (7). GA exhibits notable inhibition of proliferation, induction of apoptosis, reversion of multidrug resistance and antiangiogenesis $(6,8,9)$. Although the underlying mechanism is not fully understood, studies have demonstrated that GA induces cytotoxity in a variety of tumor cells by cell cycle arrest, interaction with the oncogene v-myc avian myelocytomatosis viral oncogene homolog and inhibition of telomerase activity (9). In addition, GA has shown to downregulate the expression of nuclear receptor coactivator 3 and inhibit the protein kinase B (AKT) pathway in leukemia K562 cells (10). Therefore, investigation into the mechanisms responsible for GA-mediated antitumor effects is likely to have great clinical significance. The present study aimed to elucidate the underlying antitumor activity of GA in ESCC and provided a novel insight into ESCC treatment. 


\section{Materials and methods}

Cell culture. ESCC TE-1 cells were purchased from the Health Science Research Resources Bank (Osaka, Japan). The cells were cultured in RPMI-1640 medium (Sigma-Aldrich, St. Louis, MO, USA) supplemented with $10 \%$ heat-inactivated fetal bovine serum (FBS; Gibco; Thermo Fisher Scientific,Inc., Waltham, MA, USA) at $37^{\circ} \mathrm{C}$ in an atmosphere containing $5 \%$ $\mathrm{CO}_{2}$.

Cell proliferation assay. GA was purchased from Sigma-Aldrich. A total of $1 \times 10^{4} \mathrm{TE}-1$ cells per well were plated in 96-well plates (Corning Incorporated, Corning, NY, USA) and cultured for $24 \mathrm{~h}$ at $37^{\circ} \mathrm{C}$ with $5 \% \mathrm{CO}_{2}$ in a humidified atmosphere. At the indicated time points $(12,24$ and $36 \mathrm{~h})$ following various concentrations of GA treatment $(2-10 \mu \mathrm{g} / \mathrm{ml})$, $10 \mu 1$ of Cell Counting Kit-8 (CCK-8; Dojindo Molecular Technologies, Inc., Kumamoto, Japan) reagent was added to each well and incubated at $37^{\circ} \mathrm{C}$ for $3 \mathrm{~h}$. Absorbance was measured at $450 \mathrm{~nm}$ in a spectrophotometer (UV-2802PCU; CRAIC Technologies, Inc., San Dimas, CA, USA). Each experiment was performed in triplicate and repeated at least two times.

Preparation of cytosolic fractions. TE-1 cells were harvested and then disrupted in lysis buffer A [0.33 M sucrose, $10 \mathrm{mM}$ Hepes (pH 7.4; Sigma-Aldrich), $1 \mathrm{mM} \mathrm{MgCl}_{2}, 0.1 \%$ Triton X-100 (Sigma-Aldrich), protease inhibitor cocktail (PIC; Sigma-Aldrich) and phenylmethanesulfonyl fluoride (PMSF)]. The cell lysates were centrifuged for $5 \mathrm{~min}$ at $800 \mathrm{x} \mathrm{g}$, and the supernatants were collected to use as the cytosolic fractions. The resulting pellets were resuspended in lysis buffer B [0.45 M NaCl, $10 \mathrm{mM}$ Hepes (pH 7.4), PIC and PMSF] and centrifuged for $5 \mathrm{~min}$ at $18,000 \mathrm{xg}$. The supernatants were collected to use as the cytosolic fractions. Samples were frozen in aliquots in liquid nitrogen and stored at $-80^{\circ} \mathrm{C}$. PMSF, sodium orthovanadate, sodium fluoride and PIC were purchased from Roche Diagnostics (Indianapolis, IN, USA).

Apoptosis assays. Apoptosis was determined by terminal deoxynucleotidyl transferase dUTP nick end labeling (TUNEL) assay, using an In Situ Cell-Death Detection kit (Boehringer Mannheim; Roche Diagnostics), according to the manufacturer's protocol. Briefly, 5,000 TE-1 cells/well were treated with various concentrations $(0,4,6,8$ and $10 \mu \mathrm{g} / \mathrm{ml})$ of GA for $24 \mathrm{~h}$ in $96-w e l l$ plates (Corning Incorporated). Following treatment, cells were trypsinized (Thermo Fisher Scientific, Inc.) and cytospin $\left(9,000 \mathrm{x}\right.$ g at $4^{\circ} \mathrm{C}$; Eppendorf 5810R; Eppendorf, Hamburg, Germany) preparations were obtained. Cells were fixed with freshly prepared paraformaldehyde (Thermo Fisher Scientific, Inc.) [4\% in phosphate-buffered saline (PBS; Thermo Fisher Scientific, Inc.); pH 7.4], rinsed with PBS three times (5 min each time), and incubated in permeabilization solution (Sigma-Aldrich). Subsequent to cross-reaction with the TUNEL reaction mixture for $60 \mathrm{~min}$ at $37^{\circ} \mathrm{C}$ and cross-reaction with converter-alkaline phosphatase solution (Sigma-Aldrich) for $30 \mathrm{~min}$ at $37^{\circ} \mathrm{C}$ in a humidified chamber, the slides were reacted with alkaline phosphatase substrate solution for 5-10 min (Vector Laboratories, Inc., Burlingame, CA, USA), rinsed with PBS three times (5 min each time) and mounted under a coverslip for analysis with a light microscope
(CX23; Olympus Corporation, Tokyo, Japan). The number of TUNEL-positive cells was assessed at X40 magnification, and representative fields were photographed (using a Nikon L200 camera; Nikon Corporation, Tokyo, Japan). The percentages of apoptotic cells were calculated from the ratio of apoptotic cells to total cells counted. A minimum of 500 cells was counted in five isolated fields, and assays were performed in duplicate, three times (six times in total). An AKT selective inhibitor, GSK690693, was purchased from Selleck Chemicals (Houston, TX, USA).

Caspase activity assay. The activity of caspase- 3 was measured using a caspase-3 assay kit (\#KA0740; Abnova, Walnut, CA, USA), according to the manufacturer's protocol. Briefly, TE-1 cells were treated with various concentrations $(0,4,6$, 8 and $10 \mu \mathrm{g} / \mathrm{ml}$ ) of GA with or without z-VAD for $24 \mathrm{~h}$, and then the cell lysis buffer containing $50 \mu \mathrm{g}$ of protein was incubated with $5 \mu \mathrm{l}$ of $4 \mathrm{mM}$ peptide nucleic acid (pNA)-conjugated substrate at $37^{\circ} \mathrm{C}$ for $2 \mathrm{~h}$. The amount of pNA released was measured at $405 \mathrm{~nm}$ using a spectrophotometer (UV-2802PCU; CRAIC Technologies). Samples without cell lysates or substrates acted as controls. All measurements of caspase activity were performed in duplicate and only samples in which the differences between the values obtained across replicates did not exceed $10 \%$ were used to calculate the mean value.

Western blot analysis. Culture cells were lysed in cell lysis buffer containing phosphatase inhibitor cocktail and proteinase inhibitor cocktail (Sigma-Aldrich), and the protein concentrations were determined using the BCA Protein Assay kit (Pierce Biotechnology, Inc., Rockford, IL, USA). Total protein (20-40 mg) was subjected to sodium dodecyl sulfate-polyacrylamide gel electrophoresis (Bio-Rad Laboratories, Inc., Hercules, CA, USA) under reducing conditions and transferred to polyvinylidene difluoride membranes (Bio-Rad Laboratories, Inc.). The membranes were blocked with Tris-buffered saline (Sigma-Aldrich) containing $0.05 \%$ Tween-20 (Sigma-Aldrich) and either 5\% skim milk or 5\% bovine serum albumin (Sigma-Aldrich), and incubated with monoclonal rabbit antibodies against NF- $\kappa B$ (\#4764; dilution, 1:1,000), AKT (\#8596; dilution, 1:1,000) and phosphorylated-AKT (p-AKT; clone Ser473; \#4060; dilution, 1:1,000), which were all purchased from Cell Signaling Technology, Inc. (Danvers, MA, USA). Subsequent to washing three times with PBS, the membranes were incubated for $1 \mathrm{~h}$ at room temperature with species-specific horseradish peroxidase-conjugated goat anti-rabbit immunoglobulin G secondary antibody (\#7074; dilution, 1:2,000; Cell Signaling Technology, Inc.). Immunoreactive bands were visualized using SuperSignal West Dura Extended Duration Substrate Enhanced Chemiluminescent Substrate (Pierce Biotechnology, Inc.). Each experiment was performed at least 3 times independently.

Autophagy assay. Monodansylcadaverine (MDC) is also a specific marker for autophagic vacuoles. To confirm that GA treatment induces autophagy, TE-1 cells were treated with various concentrations $(0,4,6,8$ and $10 \mu \mathrm{g} / \mathrm{ml})$ of GA for $24 \mathrm{~h}$. The autophagic vacuoles were labeled with MDC by incubating with $0.05 \mathrm{mM} / 1 \mathrm{MDC}$ (Sigma-Aldrich) in PBS at $37^{\circ} \mathrm{C}$ for $2 \mathrm{~h}$. Cells were then washed three times ( 5 min each time) 


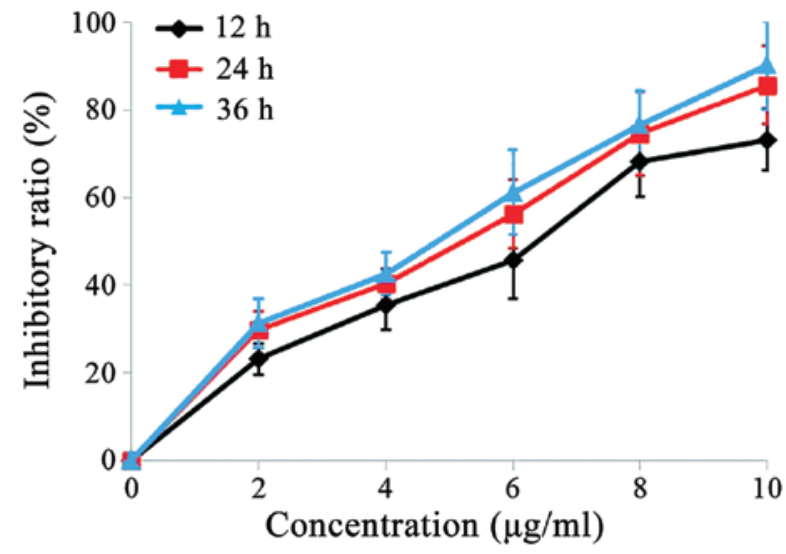

Figure 1. Inhibitory effect of GA on TE-1 cell proliferation in vitro. The cells were cultured in RPMI-1640 medium for $24 \mathrm{~h}$ and then incubated with various concentrations of GA $(0,2,4,6,8$ and $10 \mu \mathrm{g} / \mathrm{ml})$ for 12,24 and 36 h. Viability was determined by the Cell Counting Kit- 8 assay. Data are presented as the mean \pm standard error of the mean of the results for three independent experiments. GA, gambogic acid.

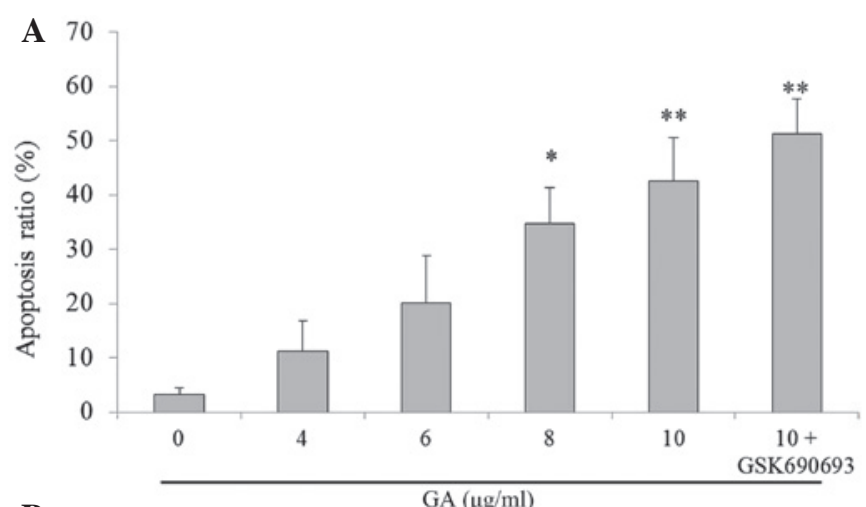

$\mathbf{B}$

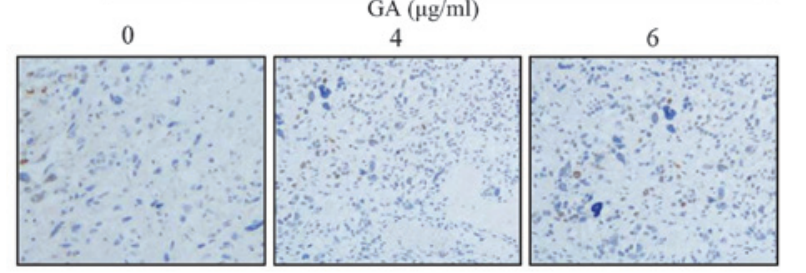

8

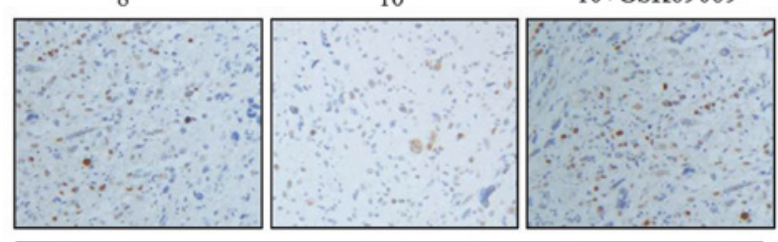

$\mathrm{GA}(\mu \mathrm{g} / \mathrm{ml})$

Figure 2. Apoptosis in TE-1 cells were induced by GA. (A) Cells were treated with various concentrations of GA $(0,4,6,8$ and $10 \mu \mathrm{g} / \mathrm{ml} \pm \mathrm{GSK} 690693)$ for $24 \mathrm{~h}$, and then subjected to TUNEL alkaline phosphatase assay. (B) The number of TUNEL-positive cells was counted in five isolated fields under a light microscope at $\mathrm{x} 40$ magnification. Percentages of apoptotic cells were calculated from the ratio of apoptotic cells:total cells counted. Mean \pm standard error of the mean; $\mathrm{n}=3$. ${ }^{*} \mathrm{P}<0.05$ and ${ }^{* *} \mathrm{P}<0.01$ vs. vehicle group (0 $\mu \mathrm{g} / \mathrm{ml}$ GA). GA, gambogic acid; TUNEL, terminal deoxynucleotidyl transferase dUTP nick end labeling.

with cold PBS buffer and immediately measured under a flow cytometer (FACScan; BD Biosciences, San Jose, CA, USA) using the Cell Quest software version 4.0 (BD Biosciences) to determine the percentage of cells undergoing autophagy that recruited MDC-positive particles.

Statistical analysis. All data and results presented were confirmed in at least three independent experiments, unless otherwise indicated. Data were expressed as the mean \pm standard error unless otherwise noted. The differences between groups were analyzed using a two-tailed Student's $t$-test and the null hypothesis was rejected at the 0.05 level. Significant differences were considered to be indicated by $\mathrm{P}<0.05$.

\section{Results}

GA inhibits TE-1 cell growth in vitro. To analyze the effect of GA on cell growth, the CCK-8 assay was used to measure TE-1 cell viability following exposure to various concentrations $(0,4,6,8$ and $10 \mu \mathrm{g} / \mathrm{ml})$ of GA for 12,24 and $36 \mathrm{~h}$. The result showed that GA significantly inhibited the growth of TE-1 cells in a dose- and time-dependent manner (Fig. 1). The maximum cell growth inhibition was evident at $36 \mathrm{~h}$ exposure to $10 \mu \mathrm{g} / \mathrm{ml} \mathrm{GA}$. The half maximal inhibitory concentrations $\left(\mathrm{IC}_{50}\right)$ of 12,24 and $36 \mathrm{~h}$ of GA treatment for TE-1 cells were $6.5,5.8$ and $5.3 \mu \mathrm{g} / \mathrm{ml}$ respectively.

GA induces TE-1 cells apoptosis in vitro. The inhibition of cell proliferation may result from the induction of apoptosis. Apoptosis rate was first observed using TUNEL analysis in TE-1 cells following treatment with various concentrations $(0,4,6,8$ and $10 \mu \mathrm{g} / \mathrm{ml})$ of GA. The result indicated that GA may significantly increase the percentage of TE-1 cell apoptosis in a dose-dependent manner (between 11.2 and 42.6\%), which may be augmented by the AKT inhibitor, GSK690693 (Fig. 2A and B).

GA promotes caspase-3 activities in TE-1 cells in vitro. Since caspase-3, a member of the family of aspartate-specific cysteinyl proteases, has been identified as a key mediator of apoptosis in mammalian cells by cleaving a variety of key cellular proteins (11), the activation of caspase-3 was investigated following treatment with various concentrations $(0,4,6$, 8 and $10 \mu \mathrm{g} / \mathrm{ml}$ ) of GA in TE-1 cells. The result showed that caspase- 3 activity may be promoted by GA in a dose-dependent manner, which may be reduced by z-VAD (Fig. 3A). Another CCK-8 assay was performed to ascertain whether z-VAD reverses GA-induced growth inhibition in TE-1 cells. The results indicated that $\mathrm{z}-\mathrm{VAD}$ attenuated GA-induced growth inhibition in the cells (Fig. 3B).

$G A$ decreases the expression of $p-A K T$ and $N F-\kappa B$ in $T E-1$ cells. Since $\mathrm{p}-\mathrm{AKT}$ and transcription factor $\mathrm{NF}-\kappa \mathrm{B}$ mainly mediate cell apoptosis, western blot analysis was conducted to ascertain the expression status of $\mathrm{p}-\mathrm{AKT}$ and $\mathrm{NF}-\kappa \mathrm{B}$ following treatment with GA in TE-1 cells. In the current study, GA was indicated to decrease the expression of $\mathrm{p}-\mathrm{AKT}$ and $\mathrm{NF}-\kappa \mathrm{B}$ in TE-1 cells in a dose-dependent manner (Fig. 4).

GA does not induce autophagy in TE-1 cells. The involvement of autophagy in GA-treated TE-1 cells was then examined. Compared with the control group, GA only caused a slight increase in the MDC positive ratio (Fig. 5). In addition, there 

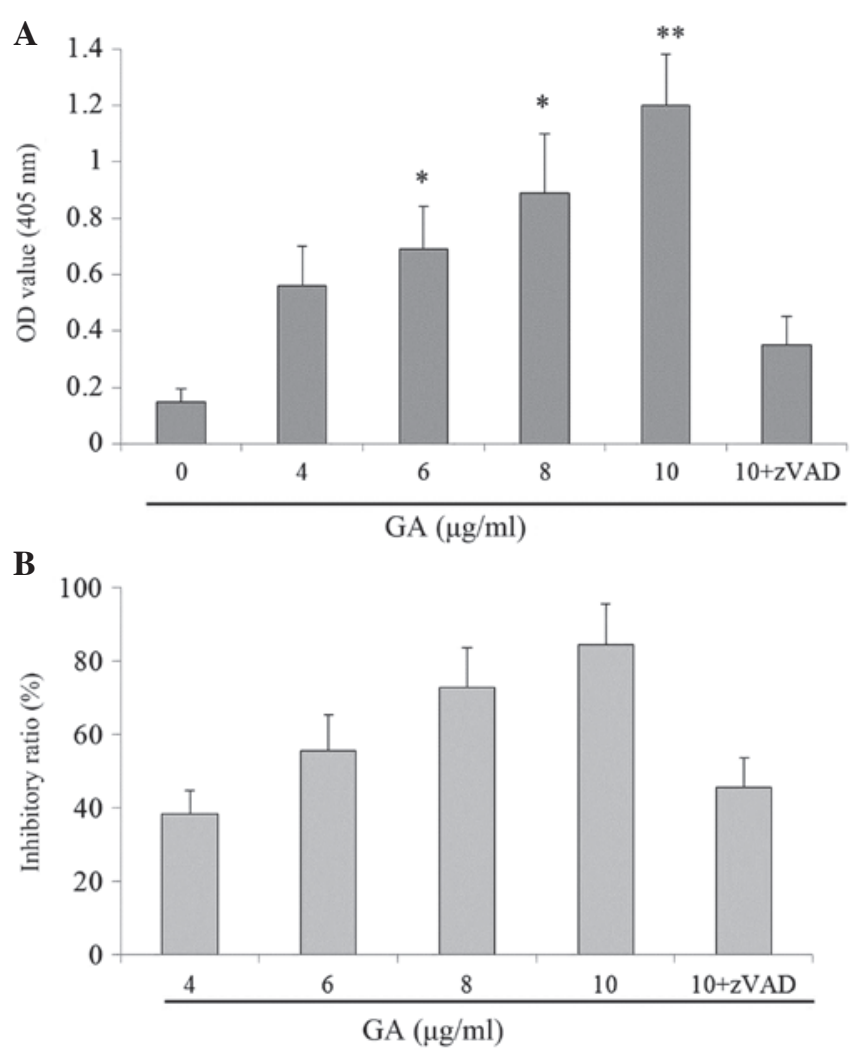

Figure 3. GA promoted caspase-3 activities in TE-1 cells in vitro. (A) Cells were treated with various concentrations of GA $(0,4,6,8,10$ and $>10 \mu \mathrm{g} / \mathrm{ml})$ for $24 \mathrm{~h}$, and then subjected to caspase-3 colorimetric protease kits. (B) Cells were cultured in RPMI-1640 medium for $24 \mathrm{~h}$ and then incubated with various concentrations of GA $(4,6,8$ and $10 \mu \mathrm{g} / \mathrm{ml})$ with or without z-VAD for $24 \mathrm{~h}$. The viability was determined by the CCK- 8 assay. Data are presented as the mean \pm standard error of th mean of the results for three independent experiments. ${ }^{*} \mathrm{P}<0.05$ and ${ }^{* *} \mathrm{P}<0.01$ vs. vehicle group $(0 \mu \mathrm{g} / \mathrm{ml} \mathrm{GA})$. OD, optical density; GA, gambogic acid; zVAD, z-VAD-fmk.

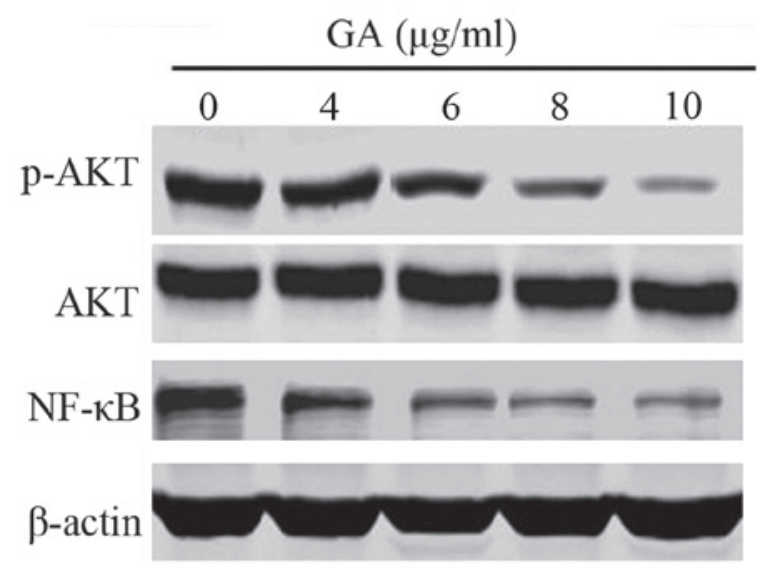

Figure 4. Inhibitory effects of GA treatments on AKT/NF- $\mathrm{BB}$ pathways. Cells were treated with various concentrations of GA $(0,4,6,8$ and $10 \mu \mathrm{g} / \mathrm{ml})$ for $24 \mathrm{~h}$. Cell lysates were then collected to detect p-AKT/AKT and NF-кB. GA, gambogic acid; AKT, protein kinase B; p-AKT, phosphorylated-protein kinase $\mathrm{B} ; \mathrm{NF}-\kappa \mathrm{B}$, nuclear factor of $\kappa$ light polypeptide gene enhancer in B-cells 1.

was no significant difference between the treatment and the control group, regarding cell morphology. The results indicated that GA may not induce autophagy in TE-1 cells.

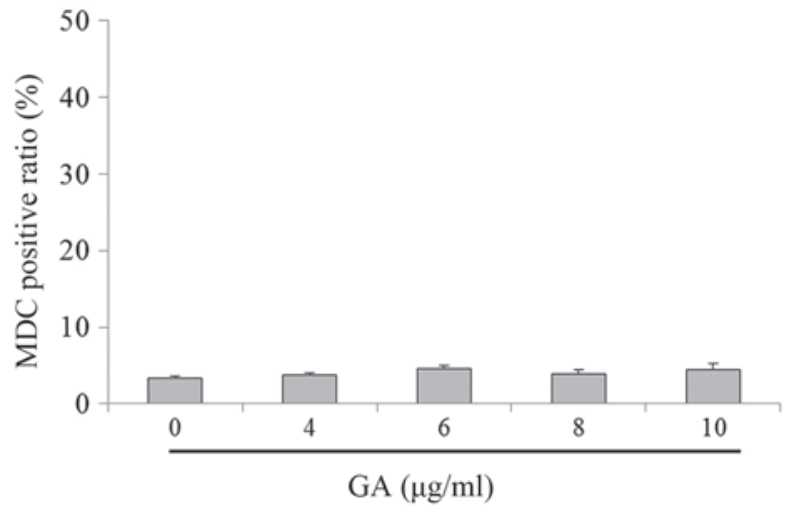

Figure 5. GA did not induce autophagy in TE-1 cells. The cells were treated with various concentrations of GA $(0,4,6,8$ and $10 \mu \mathrm{g} / \mathrm{ml})$ for $24 \mathrm{~h}$, and then subjected to quantitative analysis, which detected a positive ratio of MDC staining by flow cytometry.

\section{Discussion}

Programmed cell death (PCD), a critical mechanism for the development and homeostasis of multicellular organisms, and consists of two major forms: Apoptosis and autophagy (12). Apoptosis (type I PCD) is a cell-intrinsic suicide mechanism regulated by various cellular signaling pathways (13). Accumulating evidence has shown that GA may exert antitumor effects against a variety of human cancers cell lines, including hepatoma, breast cancer and gastric and lung carcinoma $(6,14,15)$. To the best of our knowledge, the present study is the first to suggest that GA may significantly induce TE-1 cells apoptosis. In addition, the antitumor activity of GA was accompanied by the decreased expression of $\mathrm{p}-\mathrm{AKT}$ and $\mathrm{NF}-\mathrm{kB}$, and the inhibition of AKT and NF- $\mathrm{kB}$ activation by chemical inhibitors augmented the apoptotic effect responses to GA in the TE-1 cells.

As an alternative death pathway to apoptosis, autophagic cell death has achieved the great prominence, and the mutual association between apoptotic and autophagic cell death is under debate. Luo et al reported that GA may induce apoptosis and autophagy in glioblastoma cells and that autophagy inhibition promoted apoptosis (16). Gu et al also reported that reactive oxygen species-mediated autophagy induced by the dysregulation of lipid metabolism protects colorectal cancer cells treated with GA (14). However, in the present study, the MDC positive ratio levels were not affected by GA in TE-1 cells, which indicated that GA may not induce autophagy in TE-1 cells. Shi et al reported that another natural product, shikonin, promotes autophagy in BXPC-3 human pancreatic cancer cells through the phosphatidylinositol-4,5-bisphosphate 3-kinase/AKT signaling pathway (17). It is possible that the $\mathrm{AKT} / \mathrm{NF}-\mathrm{\kappa B}$ pathway activity is also required for autophagy induction by GA in TE-1 cells (17).

TCMs have been used for more than a millennium in China to prevent and alleviate a wide variety of diseases. TCM is a key component of the multidisciplinary treatment for advanced tumors. A large number of studies showed that the proper use of TCM-based therapies may enhance immune function, speed up recovery, alleviate chemoradiotherapy-associated toxicities, improve quality of life and extend survival (18-20). 
However, the majority of TCM-based treatment only would be categorized as palliative therapy in a clinical setting. The exact antitumor mechanisms of TCM remain unclear, which hinders the usage of TCM in clinical cancer treatment. The present study demonstrates that GA may result in significant arrest of growth in TE-1 cells. In addition, GA induces apoptosis in ESCC TE-1 cells via suppression of the NF-kB pathway. The present study attempts to explain the mechanism underlying the antitumor effects of GA in ESCC, which may aid in the breakthrough of TCM in the radical treatment of malignancies.

The natural product GA is a promising novel antitumor agent that acts via various mechanisms in solid tumors and hematological malignancies. GA may be suitable for exploitation against various malignancies that are refractory to standard care as GA is indicated to act through numerous antitumor mechanisms.

\section{Acknowledgements}

The authors would like to thank Dr Ke Wu (Department of Thoracic Surgery, YueBei People's Hospital, Shaoguan, China) for providing valuable technical assistance.

\section{References}

1. Dawsey SP, Tonui S, Parker RK, Fitzwater JW, Dawsey SM, White RE and Abnet CC: Esophageal cancer in young people: A case series of 109 cases and review of the literature. PLoS One 5: e14080, 2010.

2. Siegel R, Ward E, Brawley O and Jemal A: Cancer statistics, 2011: The impact of eliminating socioeconomic and racial disparities on premature cancer deaths. CA Cancer J Clin 61: 212-236, 2011.

3. Ando N, Ozawa S, Kitagawa Y, Shinozawa Y and Kitajima M: Improvement in the results of surgical treatment of advanced squamous esophageal carcinoma during 15 consecutive years. Ann Surg 232: 225-232, 2000.

4. Strong VE, D'Amico TA, Kleinberg L and Ajani J: Impact of the 7th Edition AJCC staging classification on the NCCN clinical practice guidelines in oncology for gastric and esophageal cancers. J Natl Compr Canc Netw 11: 60-66, 2013.

5. Iwase H, Shimada M, Tsuzuki T, Hirashima N, Okeya M, Hibino Y, Ryuge N, Yokoi M, Kida Y, Kuno T, et al: Concurrent chemoradiotherapy with a novel fluoropyrimidine, $\mathrm{S}-1$ and cisplatin for locally advanced esophageal cancer: Long-term results of a phase II trial. Oncology 84: 342-349, 2013.
6. Zhao L, Guo QL, You QD, Wu ZQ and Gu HY: Gambogic acid induces apoptosis and regulates expressions of Bax and Bcl-2 protein in human gastric carcinoma MGC- 803 cells. Biol Pharm Bull 27: 998-1003, 2004.

7. Gu H, Rao S, Zhao J, Wang J, Mu R, Rong J, Tao L, Qi Q, You Q and Guo Q: Gambogic acid reduced bcl-2 expression via p53 in human breast MCF-7 cancer cells. J Cancer Res Clin Oncol 135: 1777-1782, 2009.

8. Xie H, Qin YX, Zhou YL, Tong LJ, Lin LP, Geng MY, Duan WH and Ding J: GA3, a new gambogic acid derivative, exhibits potent antitumor activities in vitro via apoptosis-involved mechanisms. Acta Pharmacol Sin 30: 346-354, 2009.

9. Guo QL, You QD, WuZQ, Yuan ST and Zhao L: General gambogic acids inhibited growth of human hepatoma SMMC-7721 cells in vitro and in nude mice. Acta Pharmacol Sin 25: 769-774, 2004.

10. Colo GP, Rubio MF, Nojek IM, Werbajh SE, Echeverría PC, Alvarado CV, Nahmod VE, Galigniana MD and Costas MA: The p160 nuclear receptor co-activator RAC3 exerts an anti-apoptotic role through a cytoplasmatic action. Oncogene 27: 2430-2444, 2008.

11. Nicholson DW and Thornberry NA: Caspases: Killer proteases. Trends Biochem Sci 22: 299-306, 1997.

12. Savill J and Fadok VA: Corpse clearance defines the meaning of cell death. Nature 407: 784-788, 2000.

13. Danial NN and Korsmeyer SJ: Cell death: Critical control points. Cell 116: 205-219, 2004.

14. Gu HY, Guo QL, You QD, Liu W, Qi Q, Li Z, Yuan ST and Zhang K: Gambogic acid inducing apoptosis in human hepatoma SMMC-7721 cells with p53 and Bax up-regulated. Chin J Nat Med 3: 169-171, 2005.

15. Xu J, Zhou M, Ouyang J, Wang J, Zhang Q, Xu Y, Xu Y, Zhang Q, Xu X and Zeng H: Gambogic acid induces mitochondria-dependent apoptosis by modulation of Bcl-2 and Bax in mantle cell lymphoma JeKo-1 cells. Zhongguo Aizheng Yanjiu (Yingwenban) 25: 183-191, 2013.

16. Luo GX, Cai J, Lin JZ, Luo WS, Luo HS, Jiang YY and Zhang Y: Autophagy inhibition promotes gambogic acid-induced suppression of growth and apoptosis in glioblastoma cells. Asian Pac J Cancer Prev 13: 6211-6216, 2012.

17. Shi SQ and Cao HM: Shikonin promotes autophagy in BXPC-3 human pancreatic cancer cells through the PI3K/Akt signaling pathway. Oncol Lett 8: 1087-1089, 2014.

18. Zhang BC: Treatment of symptoms and root causes of disease-management of the top ten symptoms in cancer patients. Fang Ai Tian Di 20: 17-18, 2007.

19. Smith ME and Bauer-Wu S: Traditional Chinese Medicine for cancer-related symptoms. Semin Oncol Nurs 28: 64-97, 2012.

20. Wang S, Wu X, Tan M, Gong J, Tan W, Bian B, Chen M and Wang Y: Fighting fire with fire: Poisonous Chinese herbal medicine for cancer therapy. J Ethnopharmacol 140: 33-45, 2012. 